\title{
Must-Have Capabilities for Enterprise Mobility: A South African Study
}

\author{
B-Abee Toperesu \\ University of Cape Town, South Africa \\ Jean-Paul Van Belle \\ University of Cape Town, South Africa
}

\begin{abstract}
Mobile devices represent a difficult information technology decision for organizations, commonly referred to as the "Bring-Your-Own-Device" (BYOD) issue. On the one hand, information is now potentially available anytime, anywhere and some business processes can be optimized dramatically. On the other hand, business processes have to be changed, security issues have to be addressed, and data management and governance become very difficult. This study presents some empirical research based on interviews with selected IS Managers. The goal of this paper is to assess both a 'desired state' of capabilities as well as some evidence regarding current readiness and capability levels among South African organizations. We conclude with a proposed list of the must-have capabilities for Enterprise Mobility.
\end{abstract}

Keywords: Enterprise Mobility, ICT, Innovation Capability, Bring Your Own Device (BYOD), Mobile Device Management

JEL classification: $\mathrm{O} 33$

Acknowledgments: This research was financially supported by the National Research Foundation (NRF) of South Africa.

\section{Introduction}

Mobile technology has advanced, bringing forth new possibilities. There have been considerable developments in mobile hardware, software and networks that contribute greatly to mobile collaboration and communication. Mobile hardware available on the market includes tablet computers and smartphones, while advanced software platforms have been developed for these devices such as iOS and Android. Advanced mobile networks such as $3 G$ and LTE allow seamless connection of these devices with fast data transfer rates enabling advanced communication software such as Skype video calling to work seamlessly. These advancements in mobile technologies have made mobile devices sophisticated and powerful to the extent that their performance nearly equals that of desktop computers (Ghosh, Gajar, \& Rai, 2013).

The traditional workplace is changing as individuals now perform their work duties using mobile devices at home, from remote sites or while on the go. Developments in mobile technologies have enabled workers to work and collaborate while on the move (Kietzmann et al., 2013). However, enterprise mobility has not received much research to date (Sørensen, 2014).

The purpose of this research study is to come up with an empirically validated list of 'must-have' capabilities for enterprise mobility. Qualitative data was collected and analyzed and final conclusions drawn from it. 
This study seeks to contribute new insights on enterprise mobility to both theory and practice. Organizations seeking to optimize enterprise mobility can use the research findings as a reference. Researchers may find the proposed model of factors, capabilities and impacts useful for research into enterprise mobility and related fields.

The paper first presents a literature review outlining the benefits, issues and impacts of enterprise mobility; and introduces the theoretical framework. It then discusses the methodology, followed by a detailed analysis of the empirical data. It concludes with recommendations, limitations and suggestions for future research.

\section{Literature Review}

The key terms used in this research are Capability and Enterprise Mobility. Several definitions of capability exist depending on the context in which it is being used (Harris, 2007). This research followed the definition that "organizational capability refers to an organizational ability to perform a coordinated task, utilizing organizational resources, for the purpose of achieving a particular end result" (Helfat, 2003, p.1).Enterprise mobility is when work interaction is mobilized using mobile and ubiquitous information technology (Sørensen, 2014). Enterprise mobility can therefore be defined as "the use of mobile IT for the accomplishment, coordination and management of organizational activities"(Sørensen, 2011, p. 476).

\section{Enterprise Mobility Benefits}

There are benefits to the organization, which include cost savings, competitive advantage, a high return on investment etc. Benefits to the employee include flexibility, increased productivity, etc. While the customer or client benefits include convenience and efficiency. Basole (2007) argues that there are five types of enterprise mobility benefits: strategic benefits, informational benefits, transactional benefits, enterprise transformation, and business benefits. Table I below describes these five types of benefits in detail.

Table 1

Enterprise Mobility Benefits

\begin{tabular}{|c|c|}
\hline Type of Benefit & Details \\
\hline $\begin{array}{l}\text { Strategic } \\
\text { Benefits }\end{array}$ & $\begin{array}{l}\text { Strategic benefits include the ability to create competitive advantage, align } \\
\text { business strategies to directly support organizational goals, provide new products or } \\
\text { services, and improve relationships with customers. }\end{array}$ \\
\hline $\begin{array}{l}\text { Informational } \\
\text { Benefits }\end{array}$ & $\begin{array}{l}\text { Informational benefits include faster and easier access to internal and external } \\
\text { information, more useful, accurate and reliable information, and increased } \\
\text { flexibility for manipulation of content and format of information. }\end{array}$ \\
\hline $\begin{array}{l}\text { Transactional } \\
\text { Benefits }\end{array}$ & $\begin{array}{l}\text { Transactional benefits include operational and cost savings; supply chain } \\
\text { management savings; staff cost savings; and improved business efficiency of } \\
\text { employees, business processes, and financial resources. }\end{array}$ \\
\hline $\begin{array}{l}\text { Enterprise } \\
\text { Transformation }\end{array}$ & $\begin{array}{l}\text { Benefits associated with enterprise transformation include improved skill levels, new } \\
\text { business plans and business models, expanded capabilities, and improved } \\
\text { structure and processes. }\end{array}$ \\
\hline $\begin{array}{l}\text { Business Value } \\
\text { of ICT }\end{array}$ & $\begin{array}{l}\text { An overarching indicator of the value of ICT to the organization, which combines } \\
\text { strategic benefits, informational benefits, transactional benefits and enterprise } \\
\text { transformation benefits. }\end{array}$ \\
\hline
\end{tabular}

Source: Basole, 2007

Enterprise Mobility Impacts

EM can have a number of impacts on business; positive impacts may also be termed benefits. 
A sharp increase in employee productivity may perhaps be the greatest impact an organization may experience, with $75 \%$ of enterprises adopting EM reporting increased employee productivity (Kumar, 2012). Tech savvy employees working from mobile devices are more likely to continue working outside of work hours and thus in turn have quicker turnaround times on their assigned tasks (Pillay et al., 2013).

Direct access to inventory levels and pricing, ability to access and update customer profiles, and carrying out transactions in real-time enables elimination of redundant procedures which in turn shortens sales cycles and increases sales(Ortbach, Brockmann \& Stieglitz, 2014). Using ERP mobile applications optimizes real time access to customer orders and inventory levels for sales staff while management has access to time critical performance indicators through dashboards.

Cost savings, and thus an increased Return on Investment (ROI) can be realized by an organization when a shift from purchasing expensive desktop computing equipment is replaced by cheaper mobile devices (Basole, 2007). Significant reductions in IT support costs can also be realized when employees use their own devices. Employees would then manage their own devices or get support from their vendor, which in turn reduces IT administration by the organization.

EMM also impacts on various intangible aspects such as employee and customer satisfaction, although measuring such impacts has proven to be difficult due to a lack of assessment tools (Vuolle, 2011). However, it is acknowledged that some positive factors in EM do contribute to the overall satisfaction of the user or customer. Carden (2007) argues that the ability to check the status of a request using a mobile application like an ERP app increases customer assurance and overall satisfaction. When a customer of an insurance company is able to file a claim using a mobile app, the result is process improvement and increased efficiency as well as customer satisfaction (Giessmann, Stanoevska-Slabeva, \& de Visser, 2012).

\section{Enterprise Mobility Challenges}

As smartphones and tablets become common at the workplace, securing corporate information on these devices has become a great concern to enterprises. The main challenge is to put in place appropriate security policies while exploiting the usability of consumer applications (Sammer, Brechbühl, \& Back, 2013).

Another challenge is lack of control of the device. When the employee owns the device, it is up to them to keep the device physically secure from use or access by others and, in the event that the device is lost or stolen, the device will become a security threat to the organization. IT practices and policies, password protection and remote wipe need to be enforced; they can also be intentionally averted by employees for various reasons (Pillay et al., 2013).

Developing compatible applications has become a headache for developers trying to produce applications that work on as many mobile devices as possible. Fragmentation of mobile device platforms presents challenges such as app support, enforcing security updates, monitoring and provisioning. In an attempt to address this issue, some vendors are offering cross platform enterprise app stores as a solution since they only need a web browser (Balakrishnan, Aziz, \& Ibrahim, 2015).

\section{Conceptual Model for Organizational Capabilities}

This research used the Technology-Organization-Environment (TOE) framework (Tornatzky\& Fleischer, 1990)to identify the capabilities for improving enterprise mobility (Figure 1). The model has three components. The first component isfactors that influence certain capabilities. The factors are grouped under three contexts i.e. 
technological factors, organizational factors and environmental factors. These factors would have a positive or negative effect of the capabilities listed in the middle section. The second component is the capabilities which will be determined during data collection. These capabilities will in turn influence certain outcomes, also to be determined in this research.

Figure 1

Conceptual model for research based on the TOE framework

\begin{tabular}{|c|c|c|}
\hline Factors/Enablers (How & Capabilities (What) & Impacts/Outcomes (Why) \\
\hline $\begin{array}{l}\text { Technology: Systems; } \\
\text { Service Providers } \\
\text { Organisation: } \\
\text { Lack of HR; Culture; } \\
\text { Mgt Support; Aware- } \\
\text { ness; Size; Governance } \\
\text { Environment: } \\
\text { Regulations; Suppliers; } \\
\text { Industry; Characteris- } \\
\text { tics: Competitors }\end{array}$ & $\begin{array}{c}\text { TO } \\
\text { BE } \\
\text { DETERMINED }\end{array}$ & $\begin{array}{c}\text { Customer Experience } \\
\text { Return on Investment } \\
\text { Competitive } \\
\text { Advantage } \\
\text { Increased Sales } \\
\text { More efficient } \\
\text { operations }\end{array}$ \\
\hline
\end{tabular}

Source: Tornatzky and Fleischer, 1990

\section{Research Methodology}

The main research question is "What are the must-have capabilities for enterprise mobility?" The secondary questions are:

1. What are the Technology, Organizational and Environmental factors that influence enterprise mobility?

2. What impacts or outcomes does enterprise mobility have?

This research study is qualitative and used a post-positivist philosophy. This research was cross sectional. Our population is large organizations, which befits the term enterprise. Our findings will not necessarily be generalizable to small and medium-sized enterprises.

Five organizations were selected from each of the following sectors: Insurance, Accounting and Auditing, Whole Sale/Retail Industry. The reason of this selection is for diversification. Purposeful sampling was used in selecting these organizations in order to find senior managers who understood the study phenomenon best. Data was collected using semi-structured interviews. Questions were prepared beforehand but served as a guideline so as to cover the entire required topic field. New questions emerged during the conversation, giving the interviewee an opportunity to further elaborate their responses. Open-ended questions were used to allow the interviewee to fully express him or herself thereby providing rich data.

Interviews were recorded and transcribed; thereafter, thematic analysis and coding was used to uncover themes and patterns in the data. Thematic analysis makes use of coding themes from the data using NVivo. NVivo is a qualitative data analysis tool, which facilitates shaping, and making sense of unstructured data by producing trends and enabling hypothesis construction and demonstration.

Candidates participated in this research on a voluntary basis. The details of participants and their organizations were treated as highly confidential and aliases are used where necessary. 


\section{Data Analysis}

Interviews were conducted with 6 senior IS Managers (referred to as P1 to P6). The data was transcribed and analyzed using thematic analysis. The following section presents the themes that came up from the interviews grouped into the categories of Factors, Capabilities and Impacts.

\section{Adoption Factors}

From our theoretical framework, we have technological, organizational and environmental factors. The following are the themes that came up under each section.

The existing themes that were originally listed in the theoretical framework were technical systems and service providers. However, during the analysis none of these themes came up. Instead the theme that came up under technology was hardware as mentioned by one participant: "So you need hardware with good battery life, good connectivity options, WiFi, 3G, and as I have mentioned the right form factor screen size to be able to work" (P1). This assertion suggest that there is a need to have the right tools in place in terms of the required hardware that will run, maintain, support and manage the mobile devices.

The findings suggest that organizational culture seemed to be a big issue across all the participants" organizations as stated by the participants; "I think the most important factor is the internal cooperate culture" (P1). "People are very used to having offices, having computers and sitting doing work. Now we are telling them listen forget about that" (P2). "Some people are very paper driven so they don't... almost have resistances to change..." (P5). However, other participants did not agree as they state that users already have a culture of technology. "A lot of the users (at...) already have a culture of technology" (P4). Organizational culture then seems to be a problem to older generation of staff members who may struggle to embrace new technologies.

The only environmental theme that emerged is government regulations, although this does not seem to affect enterprise mobility much as mentioned by the participants: "Basically the factors we will have to consider is that also the POPI act is one factor (that is the Protection of personal information) so we can't, we cannot just have e-mail on the phone with normal technology because there is no security measures put in place. So we have to consider the structured and unstructured data how it's handled how it's shared how it's stored where it's stored and those kind of things yah" (P4).However participant 1 did not agree as he states that, "Those can be complied with and still have mobility as a big aspect of our business so there is no regulations as far as I know or any other factors really that affects Us" (P1).

\section{Capabilities}

This section presents the major capabilities that emerged during the interview process, based on conversation density.

At the core of enterprise mobility is connectivity. Without connectivity the whole concept of enterprise mobility is disabled as emphasized by this participant: "I think one of the biggest challenges that we have experienced is the connectivity so when people are trying to work offsite or remotely and there is a slow or unstable connection back to the office that causes the biggest issues so then people aren't able to work...So that's the single biggest issue or barrier to enterprise mobility for us (P1).

The ability to have the right hardware for enterprise mobility came out as one of the dominant themes. This includes the mobile devices themselves and their ability to connect to a network as mentioned by the participants. "Second most important is 
the hardware itself. So you need hardware with good battery life, good connectivity options, WiFi, 3G, and as I have mentioned the right form factor screen size to be able to work" (P1). "Laptops that we issue [have] built in $3 \mathrm{G}$ cards and we supply the users with sim cards and make sure you are always connected to the internet (P1).

Most participants agreed that in order to have buy-in for enterprise mobility from users, you need to have a change management strategy. Most organizations are dealing with people who are used to their old way of thinking and working, and when new ways of doing the same tasks are introduced, proper change management is essential to achieve the main goals and purposes of enterprise mobility as mentioned by these participants. "So I think the change management is critical. Making sure that people understand why you are doing it and it is there to make their lives easier (P2)."

The need for a proper device management setup was agreed upon by most participants interviewed. This is in line with the literature, which also placed emphasis on the need for a mobile device management setup to ensure secure enterprise mobility. The main purpose of mobile device management, as discussed by the participants, is to have a level of control on the devices' functions and usage with the purpose of ensuring the organization's systems are secured from outside threats, internal abuse and malicious activities.

The need to have secure enterprise mobility was raised by most participants. This theme also relates to the above previous discussion on Mobile Device Management as MDM works to ensure security. "If you look at South Africa and you look at Kenya, to them Enterprise mobility is not something that they discussed because they believe everyone to be mobile and people in organizations has mobile but are they mobile secure? Can they do remote wipe?" (P6). "Enterprise mobility it's about basically securing information on mobile devices" (P4).

Most participants feel there is a need to have mobile applications made specifically to run on mobile devices. This may include optimizing the current applications such as e-mail to run on mobile.

Most participants placed emphasis on choosing the right use cases as being very important. "You need to understand where they need mobility and what they get. You need to have a requirement, and that requirement needs to be understood" (P6). "But I think more importantly on all those is picking the right use cases I think is what you call them. Start their first because the danger is you start with the technology and then you look at the processes" (P2). Letting technology drive mobility has a negative impact of having the systems and applications which do not really serve any purpose. This would in turn affect the usability of the technology.

\section{Impacts}

This section presents a list of the impacts, outcomes and benefits that are as a result of an organization's enterprise mobility.

Most participants were of the view that, to them, enterprise mobility has brought a significant increase in productivity. "I think people have become more productive because of mobility" (P3). "What Enterprise mobility on the mobile devices has enabled us to do is to increase productivity" (P1). "I think the biggest impact is got to be on store productivity" (P2). This is in line with findings from the literature, which listed increased productivity as a major benefit. Increased productivity is due to the flexibility that comes with working from anywhere.

Increased efficiency or effectiveness also came up in the interviews. Participants mentioned that with mobility comes efficiency in doing and carrying out tasks, as emphasized by participant 5: "I mean that was the main thing to be a little more 
efficient" (P5). This is in line with the literature findings that enterprise mobility allows work to be done more efficiently, resulting in significant time savings.

Another benefit is increased sales. "So there is a full impact across the system. It starts with store efficiency and productivity that actually leads to increase in sales" (P2). It is worth noting that the increase in sales is as a result of the other impacts like efficiency and productivity as mentioned by the participant.

\section{Summary of Findings}

The data analyzed from the interviews provided the researchers with the following findings. Most of the factors that were provided in the theoretical framework were not validated by the data. However, several new factors were uncovered (Figure 2).

Under technological factors, technology systems and service providers were not validated by the data. However, hardware came up as a new theme and is an important factor as emphasized by participants. The hardware referred to includes the file servers and the mobile devices themselves that lie at the root of mobility.

Figure 2

Final organizational mobility capability model

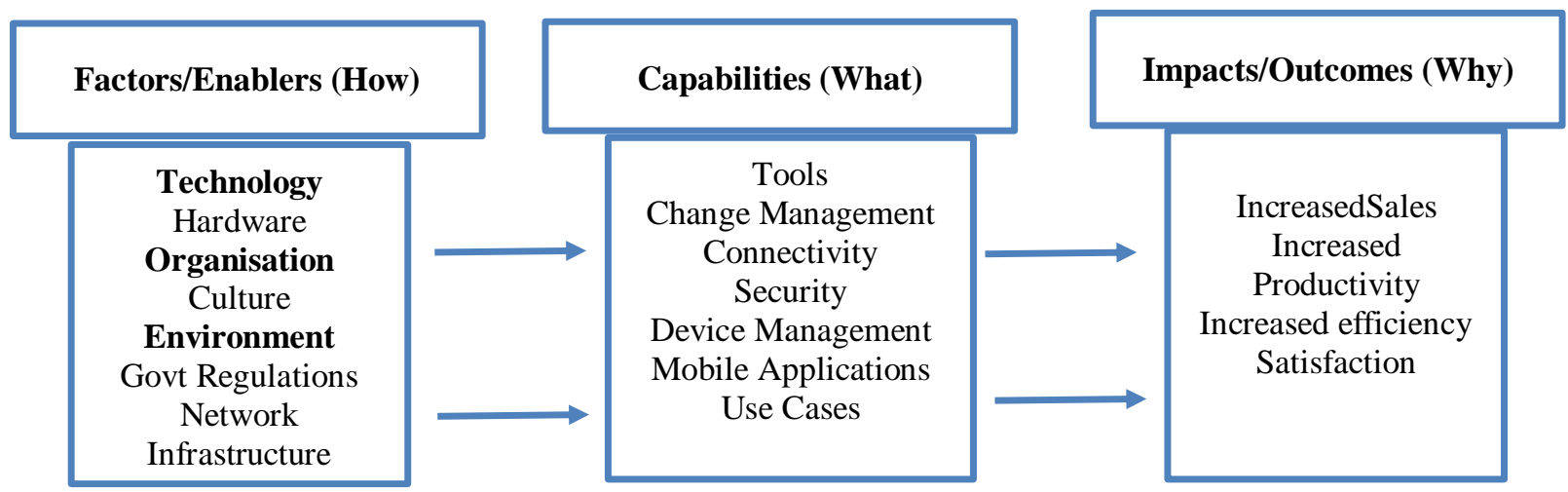

The organizational factor that affects enterprise mobility the most is culture. The other factors were not validated. However, it can be noted that lack of awareness is closely associated with culture as raised by participant 4: "It's a matter of how to do those things and there is always requests from users to see how they can counter from mobile devices" (P4).

The findings show that under environment factors, only two factors were considered to affect enterprise mobility: government regulations such as the POPI act and a new theme network infrastructure. Interestingly, the other factors originally identified were not mentioned, such as suppliers and competitors.

Capabilities were identified from the interview data. Some capabilities themes came out dominant and were in line with the literature. Although Use Cases and Change Management did not receive many references in the interviews, the emphasis that was placed on them is of much significance.

Taking a look at impacts, the findings reveal four main impacts as a result of enterprise mobility. These are satisfaction, increased sales, productivity and efficiency. User satisfaction and increased productivity were new themes that came up. The major impact highlighted by participants was increased productivity. This is due to the ability to work from 'anywhere'. Other impacts such as customer experience, return on investment and competitive advantage were not validated. 


\section{Conclusions}

The purpose of this research was to determine the "must-have" capabilities for enterprise mobility. We also investigated what Technology, Organization and Environment factors influence enterprise mobility and what impact or outcome enterprise mobility has.

The must-have capabilities for enterprise mobility which were uncovered in our empirical study are:

- Security- the ability to secure information being transmitted to and from mobile devices

- Mobile Device Management- the ability to manage the mobile devices issued to staff. This includes the remotely wiping of the devices.

- Choose the right use cases- an organization should be able to pick the right use cases on which services and processes to offer on mobile devices.

- Change Management- the ability to effectively manage the change in shift to enterprise mobility.

- Connectivity- the ability to offer or operate in an environment where the mobile devices could be connected to a network.

In answering the secondary questions, the following findings were discovered. The factors that affect enterprise mobility are: hardware availability, culture and/or awareness, government regulations and network infrastructure. The most important impacts or outcomes of enterprise mobility to an organization are user satisfaction, increased sales, productivity and efficiency.

The research findings are of significant importance to organizations seeking to either adopt an EM strategy or to organizations currently employing an EM strategy but needing to improve it. This list of must-have capabilities acts as a reference point to organizations as to what is it they need for EM.

The major limitation for the research was the small sample size. A larger sample size might have provided much richer data and possibly revealed other factors, capabilities and impacts. Also, since most organizations were mainly based in South Africa, the findings might not be a truly indicative of all organizations worldwide.

Future research may wish to look at a particular industry in an effort to have a more accurate set of capabilities for enterprise mobility for that particular industry type. Also, an extension of this research in other country contexts is necessary in order to determine the generalizability of our findings.

\section{References}

1. Balakrishnan, N., Aziz, M., Ibrahim, F. (2015). "Enterprise App Store for Cross Platforms". IJFCC, Vol. 4 No. 2, pp. 147-151.

2. Basole, R. (2007). "Strategic Planning for Enterprise Mobility: A Readiness-Centric Approach". In AMCIS 2007 Proceedings (pp. 1-14). Association for Information Systems.

3. Basole, R. C. (2008). "Enterprise mobility: Researching a new paradigm". Information Knowledge Systems Management, Vol. 7 No.1, pp. 1-7.

4. Carden, P. (2007). "Enterprise mobility". Enriching Communications.

5. Ghosh, A., Gajar, P. K., Rai, S. (2013). "Bring Your Own Device (BYOD): Security Risks and Mitigating Strategies". Journal of Global Research in Computer Science, Vol 4 No.4, pp. 62-70.

6. Giessmann, A., Stanoevska-Slabeva, K., de Visser, B. (2012). "Mobile Enterprise Applications--Current State and Future Directions". In 2012 45th Hawaii International Conference on System Sciences (pp. 1363-1372). IEEE.

7. Harris, R. (2007). "Unravelling the notion of organisational capability: What do writers say it is and VET providers think it is?" University of South Australia. 
8. Helfat, C. (2003). "Stylized facts regarding the evolution of organizational resources and capabilities". In The SMS Blackwell handbook of organizational capabilities: Emergence, development, and change.

9. Kietzmann, J., Plangger, K., Eaton, B., Heilgenberg, K., Pitt, L., Berthon, P. (2013). "Mobility at work: A typology of mobile communities of practice and contextual ambidexterity". Journal of Strategic Information Systems, Vol. 22 No.4, pp. 282-297.

10. Kumar, A. (2012). "Enterprise Mobility Strategy-Should Enterprises Care?" Infosys Labs Breifing, Vol.10 No.1, pp. 35-48.

11. Ortbach, K., Brockmann, T., Stieglitz, S. (2014). "Drivers for The Adoption of Mobile Device Managemnet in Organisations". In ECIS 2014 Proceedings (pp. 1-19).

12. Pillay, A., Diaki, H., Nham, E., Senanayake, S., TAN, G., Deshpande, S. (2013). "Does BYOD increase risks or drive benefits?", The University of Melbourne. Available at http://minerva-access.unimelb.edu.au/handle/1 1343/33345

13. Sammer, T., Brechbühl, H., Back, A. (2013). "The New Enterprise Mobility: Seizing Opportunities \& Challenges in Corporate Mobile IT". AMCIS 2013 Proceedings (pp. 18).

14. Sørensen, C. (2011). "Enterprise Mobility: Tiny Technology with Global Impact on Work". Palgrave Macmillan.

15. Sørensen, C. (2014). "Enterprise Mobility". Computing Handbook. CRC Press, 2, 1-21.

16. Tornatzky, L. G., Fleischer, M. (1990). "The process of technology innovation". Lexington: Lexington Books.

17. Vuolle, M. (2011, December 19). "Measuring Performance Impacts of Mobile Business Services from the Customer Perspective". International Journal of Learning and Intellectual Capital, Vol. 8 No. 1, pp. 50-62

\section{About the authors}

B-Abee Toperesu is an Information Technology consultant and is doing his Master's degree in Information Systems at the University of Cape Town. He is currently researching on the impact mobile devices have on a student's learning experience in higher education. This paper is based on his honors research study on enterprise mobility. The author can be contacted at TPRBAB001@myuct.ac.za.

Jean-Paul Van Belle is a professor in the Department of Information Systems at the University of Cape Town and Director of the Centre for IT and National Development. His research areas are the adoption and use of emerging technologies in developing world contexts including mobile, cloud computing. His passions are ICT4D and adoption of ICTs by small organizations. He has over 170 peer-reviewed publications including 25 chapters in books and about 40 refereed journal articles. He has been invited to give a number of keynote presentations at international conferences and holds an honorary professorship at Amity University. He currently supervises almost 20 Masters and PhD students and has graduated many more. The author can be contacted at Jean-Paul.VanBelle@uct.ac.za. 